This is an invited program report. It describes an adaptation of OREGON STATE ADVANCE'S summer seminar for OSU's University Relations and Marketing division and resulting impacts in the division.

\title{
Addressing the Symbolic Dimension of Oppression through an Institutional Transformation Seminar with University Relations and Marketing
}

Annie Heck and Steve Clark, Oregon State University

Systems of oppression theory guides Oregon State University's (OSU) National Science Foundation funded institutional transformation program. A key component of the theory recognizes that systems of oppression are structured along three dimensions: the individual, the institutional, and the symbolic (Collins 1993). The individual dimension addresses how gender, race, class, etc. frame our personal biographies and the ways we participate in institutions and relationships. The institutional dimension names the systematic ways social institutions, such as higher education, structure relationships in order to maintain power and privilege or confer subordination. Finally the symbolic dimension acknowledges the impact of ideologies, especially as they take shape in language and stereotypes, in rationalizing and reproducing hierarchies. (Collins 1993). In other words, the symbolic dimension of oppression has to do with the ways we make meaning through language, symbols, images, brands, and messages.

The central activity and key innovation of OREGON STATE ADVANCE is a 54-hour summer seminar that helps administrators and senior faculty members understand how systems of oppression operate within universities and supports them in developing action plans to address concrete structural changes (such as hiring and promotion policies and practices) needed to bring about a more inclusive, equitable, and just university. OSU's University Relations and Marketing (URM) division is the central administrative unit tasked with managing the university's brand 
and messaging. In 2016 the URM leadership team participated in an ADVANCE seminar developed specifically for them. While addressing general university practices such as hiring and promotion, this seminar paid particular attention to the symbolic dimension of oppression and the ways URM could reimagine the university's messaging that could challenge stereotypes and dominant expectations and promote inclusion, equity, and justice. As far as we know, this endeavor is the first among ADVANCE-funded projects to work specifically with marketing and university relations professionals to support institutional transformation. As a result of this project, OSU's URM has made intentional changes in staffing and messaging to address structural and ideological problems rooted in the symbolic dimension of oppression.

\section{Background}

Open and intense dialogue among Oregon State University colleagues during the summer of 2015 provided a catalyst that completely changed how OSU engages in marketing and communications, resulting in far-reaching effects to advance diversity and inclusivity throughout the university community. A 2015 marketing campaign called "Beaver Nation" leveraged the concept of the school's mascot- the Beaver-to showcase the impact of OSU and its students, faculty and staff in Oregon and beyond. Web content marketing videos shared on the web and through social media and outdoor banners placed throughout Oregon State's Corvallis campus were key elements of the campaign. The objective of the campaign, led by OSU's URM division, was to create a deeper understanding that OSU as the state of Oregon's land grant university, is accountable to serve all of Oregon, including many regions throughout the state.

The original campaign included photos, videos and stories of OSU community members in their roles pursuing teaching, research, and outreach and engagement throughout Oregon and beyond. Some banners featured illustrations of a state map and world map with a single word 
above the drawings: "Ours." In fact, at the time "the Beaver Nation" campaign professed that Beavers are responsible to serve "Our" state, "Our" nation, and "Our" world.

While the campaign was well intentioned, various campus groups and individuals provided feedback that challenged the campaign. The placement of the word "ours" over the map of the state or regions of the state, for some connoted erasure of the land's history as indigenous land. Others noted the use of "ours" over a map of Africa suggested colonial ownership. Campus leaders involved in equity and justice issues explained how such images worked within symbolic dimensions of oppression and furthered stereotypes and discrimination. Out of these conversations, URM and OREGON STATE ADVANCE agreed to develop an institutional transformation seminar geared specifically toward URM leadership and their task as managers of the university's messaging.

\section{University Relations and Marketing at Oregon State University}

University Relations and Marketing leads all central communications, marketing, and public affairs functions for OSU. The division is responsible for communicating the university's brand promise of an accessible, high-quality education. Its 42 professionals include strategists, writers, media relations specialists, designers, videographers, digital communicators and event planners among others. The team collaboratively develops differentiated branding, messaging, and creative strategies to achieve outcomes among key audiences, such as prospective students, employees, and the public. In addition to URM professionals, each of the university's 11 colleges and other key divisions — such as Intercollegiate Athletics—employ marketing and communications professionals. Approximately 165 people throughout the university are involved in marketing and communications activities. 
A key part of URM's branding work is to develop and execute creative marketing campaigns, which generally last for 18 to 24 months. As part of this work, the division uses words and images that seek to create an emotional connection to audiences and compel them to take a specific action.

After the summer of 2015, URM leadership realized that the banners that had caused concern were symptomatic of a broader issue - the need to make diversity and inclusivity a more clear and primary focus of all of the division's communications and marketing work and all of its employees. Once URM leaders determined that a major reset was needed, the team decided to participate in an ADVANCE seminar with a focus on marketing and communications. In the spring of 2016, 12 URM employees - most of whom led teams and had high level responsibilities in the division - participated in the seminar. The university's vice president of University Relations and Marketing participated in a later ADVANCE summer seminar with other central administrators.

\section{The URM OREGON STATE ADVANCE Seminar}

While systems of oppression theory has been central to women and gender studies for decades, OSU was the first ADVANCE institution to center this theory as a way to challenge and change academic culture. This theory moves us beyond locating problems of discrimination within individual bad actors and into understanding discrimination as a result of systematically related, self-reproducing institutional structures and ideologies (Johnson 2017). Because systems of oppression are structured along the three dimensions identified earlier, institutional transformation requires disruption and recreation of the entire system at institutional and symbolic levels as well as personal and individual behavior and attitudes (Collins 1993). Central to this process is a deep commitment to intersectionality in analysis and action (May 2015). 
Intersectionality is a tool for analysis that considers simultaneously experienced social identities within hierarchically structured systems of power and privilege and directs action toward social justice through disruption of oppressive hierarchies and development of empowering, egalitarian, and life-affirming relationships and institutions. Systems of oppression theory provides us with a way to explain the persistence of discrimination despite individual people's good intentions. Discrimination is not simply the result of individual bigotry and bad behavior. Rather, it is the method by which social institutions and ideologies purposefully distribute power inequitably across social differences in order to reproduce and maintain the dominance of elite, heterosexual, white manhood (May 2015). Even more insidious are the ways in which these systems of oppression obscure the origins and operations of power so that the workings of power seem natural, inherent and merited (Bird 2011). Systems of oppression theory makes these workings visible and challenges the supposed inevitability of the distribution of power across social difference.

Transforming the academy, then, requires shifting power relations and restructuring institutional arrangements to create more equitable and just policies, processes, and structures and to foster more positive interpersonal interactions. Research suggests that these changes occur most successfully when interventions target the "mechanisms that produce inequality" (Morimoto, 2013). In other words, because disparities are both method and result of systems of oppression, equity can only be achieved as the structures and ideologies that generate inequity and discrimination are thoroughly and systemically transformed.

The OREGON STATE ADVANCE seminar uses critical pedagogies (see Bothwell et al. 2018 for more detail) in an intensive immersion experience to introduce participants to theories of systems of oppression, to help them apply these theories to their own work in the university, 
and to facilitate their development of actions plans to bring about institutional structural change within their spheres of influence. To address the specific responsibilities of URM, OREGON STATE ADVANCE leadership adapted the summer seminar to focus directly on issues of the symbolic dimension of oppression to help URM leaders imagine, plan, and implement change within the university's messaging. To accommodate the schedules of those in the division, the hours were spread over the spring term as a series of eight of 6-hour workshops. While we recognize the importance of the summer seminar being done across two consecutive weeks in order to develop a cohort or community of trust, the URM group already works together and we believed would have the ability to sustain the learning as they worked together outside the seminar.

One challenge of the seminar was that it included direct supervisors for some employees (which the ADVANCE team generally tries to avoid in the seminar because of the power dynamics that may affect participation). The presence of supervisors may have limited some participants in fully engaging in conversations or pointing out areas for improvement. Additionally, since the seminar had a limited number of spaces available for participants, only a small portion of the division was able to participate. In such a large division, critical mass is essential to gain traction, and so additional opportunities for other URM employees are needed.

\section{Results of the URM ADVANCE Seminar}

Following participation in the seminar, URM leaders undertook an initiative to reorganize the division. One key element of that effort was the adoption of three organizational pillars that became the foundational principles for all of the division's work - from documentary filmmaking to social media posts to executive communications. These pillars are: (1) integrated/branded marketing, communications and public affairs; (2) socially just and inclusive 
marketing, communications and public affairs; and (3) exceptional client services and account management (OSU URM).

The OREGON STATE ADVANCE seminar clearly had a major impact on URM as evidenced by the prominence of the new lens through which all work in the division is approached. The second pillar demonstrates URM's commitment to socially just and inclusive marketing, communications, and public affairs — not just for the division, but as leaders, educators and "keepers of the brand" for the entire university's marketing and communications efforts. URM staff report significant shifts in their mindsets as a creative team, now having deeper and more critical discussions about inclusion, equity, and justice in relation to almost every piece they create. Evidence for this can be seen in the way the division has responded to the feedback on the banner campaign as well as in the way new work is taken on.

As the practice of hanging banners throughout campus continues with new marketing efforts each year, URM staff now reviews banners with this new lens. The focus of banner messaging also has shifted to promote belonging and building a greater sense of community within OSU among all students, particularly underrepresented populations. Additionally, the university's marketing and design teams engage others throughout the OSU Corvallis campus community to review and give input on creative banner concepts as needed.

This outreach is not only a form of input or an attempt to receive support for creative concepts but an education for those who may have little experience with discrimination or social justice and want to have a better understanding. With greater knowledge, they, too, can help advance diversity and inclusivity at the university.

Learning by OSU marketing leaders and staff is ongoing. Attendance at lectures with national speakers, such as comic and television personality W. Kamau Bell, or noted American 
author Colson Whitehead, provide an important - and sometimes graphic and uneasy education in intersectionality and understanding of how people are often disadvantaged simultaneously by many factors, including race, economic class, gender or identity, sexual orientation and religion.

For OSU marketing and communications professionals - most of whom do not come from diverse backgrounds - understanding how intersectionality significantly affects the disadvantaged, will lead to even more honest and socially just and inclusive work. One such example is within admissions recruitment materials that speak to diverse community members and prospective students, who otherwise might not consider OSU as being a welcoming place for them to attend college.

In that regard conversations with OSU Admissions on the recruitment and retention of a diverse student population has revealed a critical need for providing prospective students with information on the breadth of resources and activities for underrepresented communities beyond mentioning the OSU's cultural centers. Spending time with Admissions professionals as well as student ambassadors resulted a very different set of recruitment brochures (Figures 1 and 2). These brochures expressed our commitment to student success and provided links to the cultural centers, other offices and clubs on campus and to supports in the local community. They also addressed the symbolic dimension of oppression through messaging that centers diverse groups on campus, avoids stereotypes, and underlines OSU's commitment to underrepresented groups. Fig. 1 Brochure created by URM in partnership with OSU Admissions directed toward prospective indigenous, Native American, Native Alaskan, Native Hawaiian, and First Nations students 


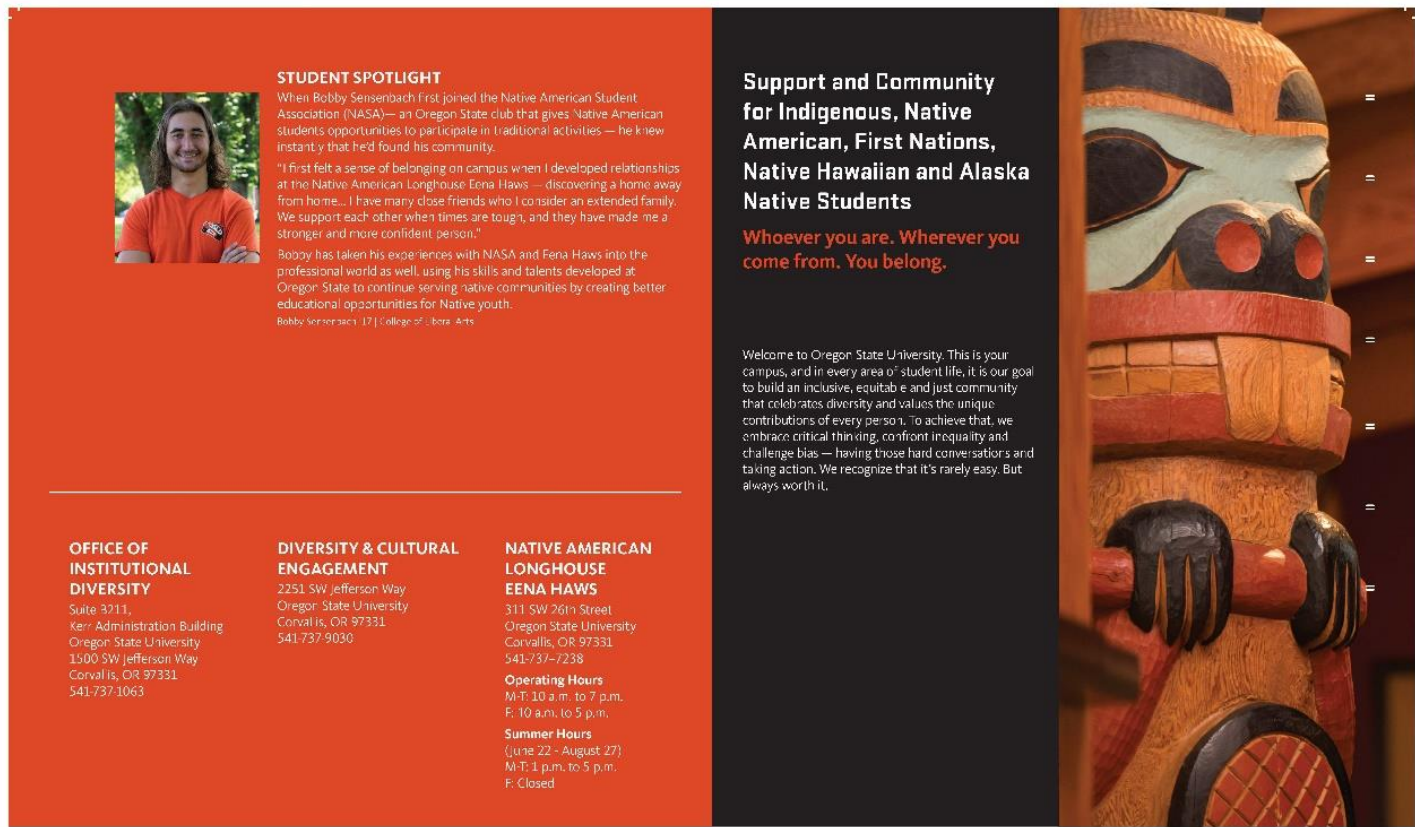

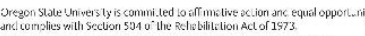

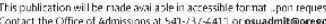

Oregon State University
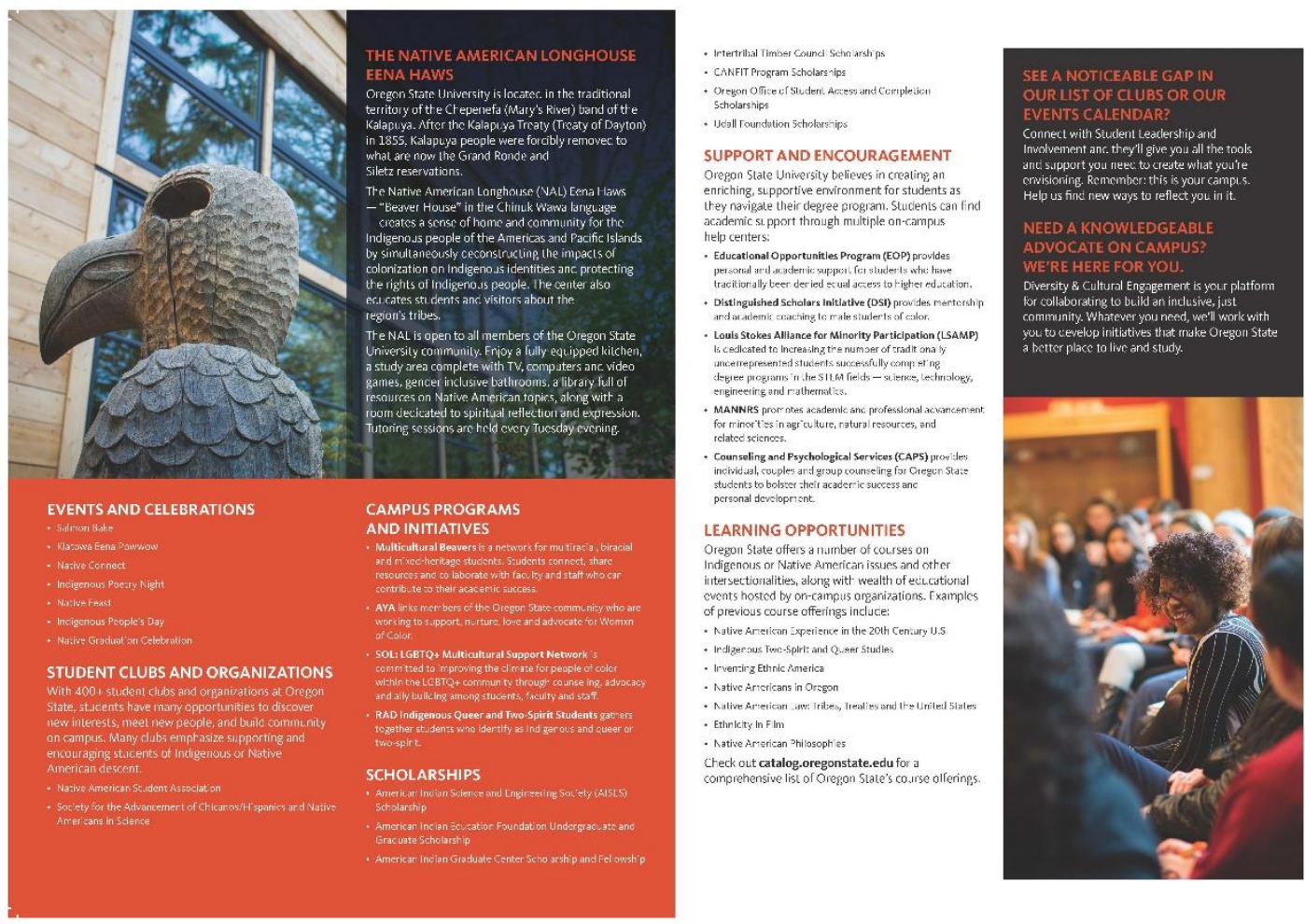

Fig. 2 Brochure created by URM in partnership with OSU Admissions for prospective LGBTQ+ students. URM created similar brochures for Chicanx/Latinx/Mestizx, Black/African/African American, Asian/Pacific Islander, and Asia/North Africa prospective students. 


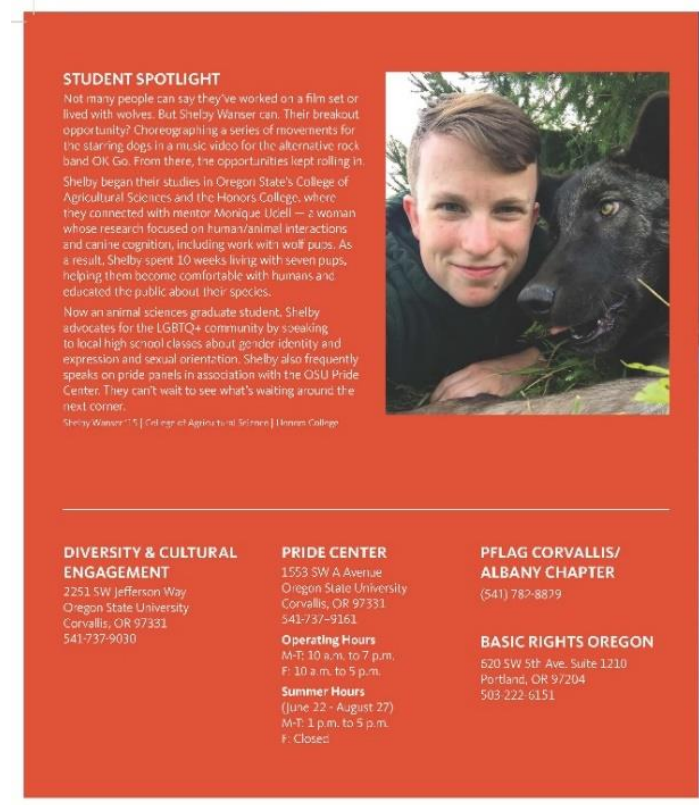

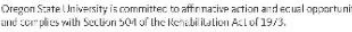

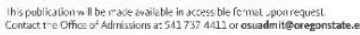

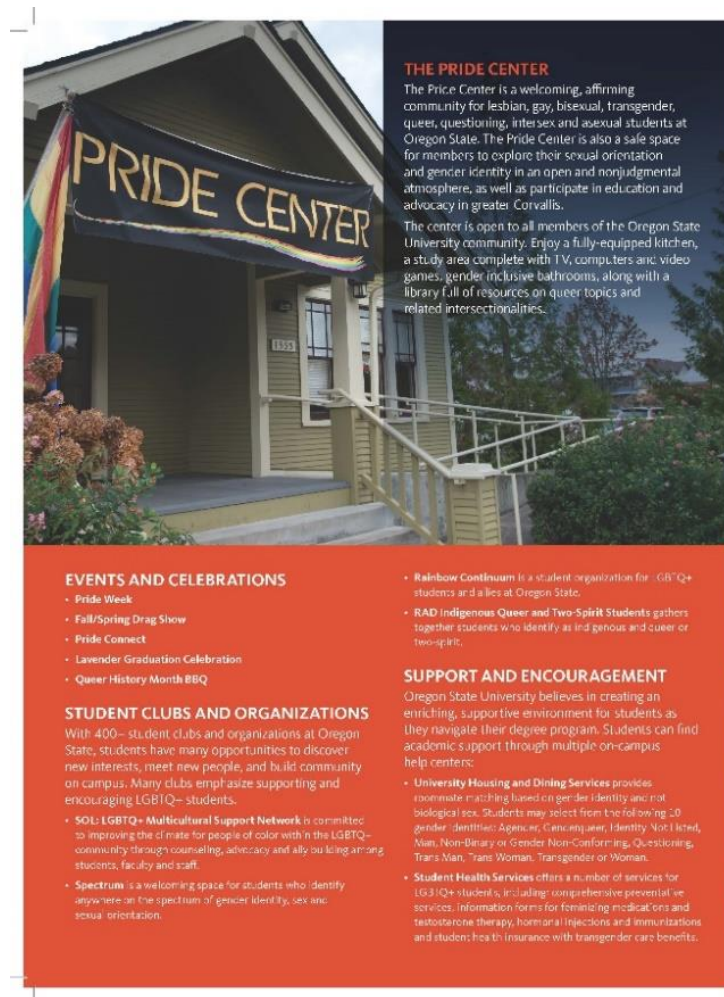

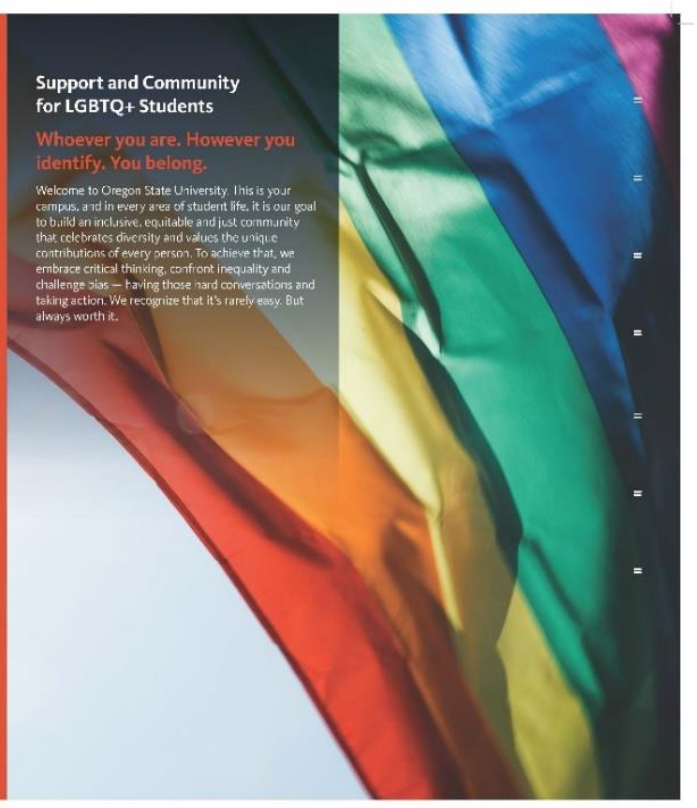

OregonState Vniversity
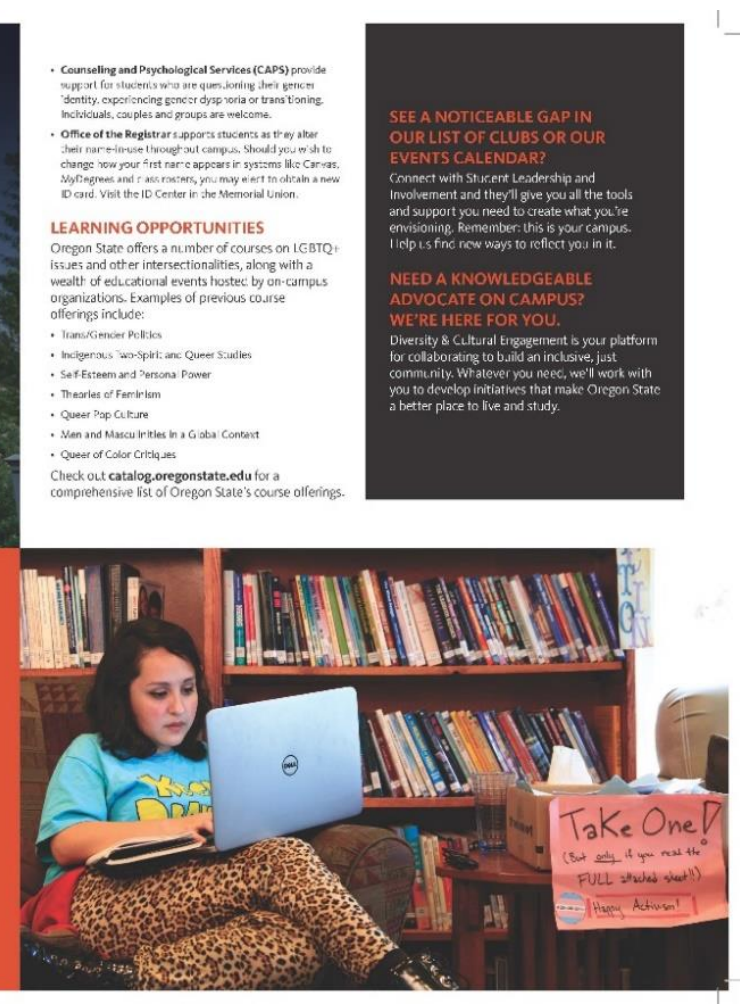
Another example of how our new lens is changing our work can be seen in a display banner for the Office of Institutional Diversity (OID) (Fig. 3). The office told URM they wanted something that visually represented the work they do but did so in an "unexpected" way. A review of marketing materials from other universities revealed that a common approach for showing support for diversity was to provide images of faculty and staff from many different communities. Often these images provide a non-representative view of a university's actual diversity. After considerable dialog with OID, URM decided to use photos of details from buildings and art on campus where conversations around diversity, equity and inclusion take place (as opposed to using photos of people). The commitment to diversity goes beyond bringing people to campus; it entails providing inclusive spaces to facilitate belonging and community. 


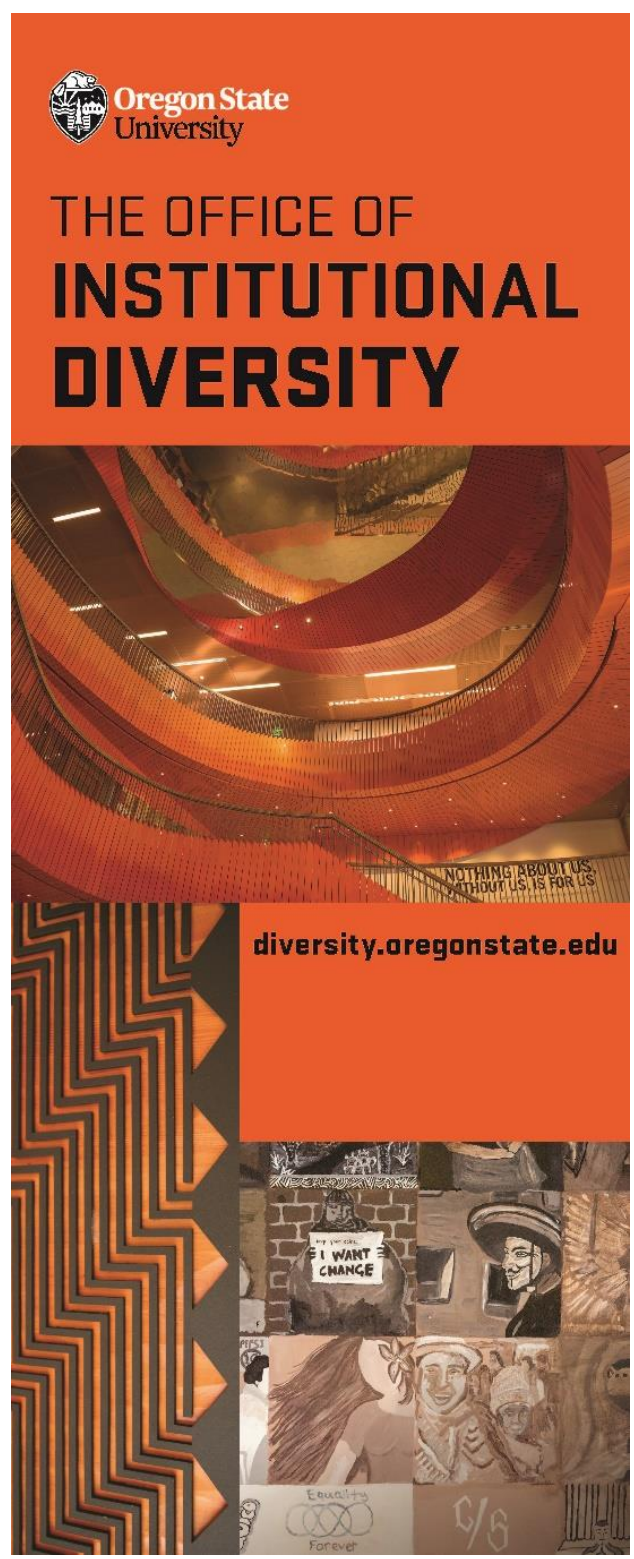

Fig. 3 URM created this display banner for the Office of Institutional Diversity, drawing from campus images of places where conversations about inclusion, equity, and justice take place.

As outlined above, a systems of oppression perspective reminds us that we must address all three dimensions for sustainable change. The URM ADVANCE seminar graduates individually took responsibility to question their own approach to work and committed to working with others across campus to develop more socially inclusive marketing. Many have participated in university workshops on implicit bias and social justice education that allow them the 
opportunity to challenge their own understandings and work to make personal change. For example, URM hiring managers and leaders working on hiring practices have agreed to have a "search advocate" from another unit on campus serve on search committees for each position that opens within the division. Established in 2008, OSU's search advocate program enhances equity and diversity in university hiring. Search advocates are OSU faculty, staff, and students trained as search and selection process advisors with the specific responsibility of providing insight into the possibilities of implicit bias in the search process. Since the summer of 2016, URM has made four new hires, all women. URM also has encouraged its employees to be trained as search advocates and become well-versed in serving the university's mission of inclusive excellence within other units' searches throughout campus. URM currently has 16 trained search advocates.

Additionally, in order to develop stronger accountability for making institutional change, the division reallocated 20 percent of the associate marketing director's time to enable a focus on managing this new priority on behalf of the division. The goal is for the associate marketing director to implement communications strategies to support the priorities of diversity and inclusion, including developing creative concepts that target diverse audiences and support Oregon State's brand image. They also provide leadership, direction, and training around strategies and trends for communicating with diverse audiences, both within University Relations and Marketing, and with other communication colleagues across the university.

To date, there have been several significant accomplishments as a result of this dedicated time and focus. In the summer of 2017, the associate marketing director worked with Academic Affairs to schedule two sessions of the Social Justice Education Initiative workshop open to all URM employees for participation. Twenty-six employees participated, including all of the 
University Marketing staff. The workshops covered topics such as historical and current social context, individual identities, zones of safety, implicit bias, micro-aggressions and cross-cultural communication. In addition, the associate director became an OREGON STATE ADVANCE Fellow. The Fellows program supports a seminar graduate for one year with the purpose of reviewing all of the individual action plans that emerged from the seminar across the Fellow's college or division. As a result of this review, Fellows have the opportunity to find alignments of individuals and work that can bring about shared efforts and greater division-wide impact. It also positions a division to know who is best positioned to work with other units across campus to achieve their marketing goals.

The URM ADVANCE fellow integrated issues of inclusion, equity, and justice throughout the unit's 2018 Brand Symposium and used their funding from the fellowship to bring nationally recognized speakers on inclusion and equity in marketing for the event. More than 150 communicators from across the university attended the symposium, and URM leaders have used the event as a launchpad to continue conversations about how inclusion, equity, and justice become more and more integral pieces of the marketing and communications work done by the university. Additionally, the fellow scheduled meetings with one of the speakers with the university's president, vice president for URM, and chief diversity officer to maximize the impact of the fellowship funding. Of course, one downside of having one colleague designated as URM's point person on inclusion, equity, and justice is that others within the unit may then rely solely on that person to provide guidance and discussion around diversity issues. Ensuring that all colleagues understand that work of inclusion, equity, and justice requires a sense of ownership from everyone in URM is essential. 
When developing new university brand guidelines in the spring of 2017, URM's senior editor worked with professionals in DCE to develop a section specifically on inclusive language practices to serve as a resource for all university communicators. Additionally, URM works with OID to tell the story of Oregon State University and its proactive efforts to advance diversity and inclusion, including creating a biannual newsletter, updating the office's website and creating marketing materials for the office. Most recently, University Marketing worked with OID to launch its "We Have Work To Do" campaign targeting students, faculty and staff (https://diversity.oregonstate.edu/we-have-work-to-do ).

One of the hallmarks of URM's journey since 2016 is the role the division has played in influencing the marketing and communications work across the university with specific focus on sharing best practices in diversity and inclusion. This was showcased at the second annual Brand Symposium. The symposium was a transformation and professional development opportunity for more than 150 attendees who heard from four national keynote speakers who addressed topics including diversity, inclusion and social justice; compelling storytelling; effective strategies for recruiting prospective students based on media trends; and finding success in creative communication pursuits. These topics directly affect the ability of marketing and communications professionals across the university to engage in activities that support undergraduate retention, recruitment of diverse faculty and students and strengthen the impact of OSU throughout Oregon and beyond.

This evolving URM culture also has expanded beyond the university's marketing and communications functions. OSU's Printing and Mailing division, the LaSells-Stewart Center-a major event venue on campus - and Conference Services have now become part of the broader University Relations and Marketing team. Since this transition, employees in these three 
additional university functions also have embraced the URM pillars and use these foundational principles to guide their work.

As often happens in institutional transformation efforts, the URM seminar provided a moment in time for participants to have a transformative experience, but keeping the momentum and energy from the seminar over a long period of time is exteremely difficult. Some of the prioritization of diversity issues has diminished over time at the division level, and enactment of some strategies and tactics in the pillar document have stalled. Our experience points to the need for efforts to sustain momentum to be explored and developed in collaboration with OREGON STATE ADVANCE.

\section{Conclusion}

URM's collaboration with OREGON STATE ADVANCE has resulted in a collective awakening among the division's leadership, which has motivated URM employees to understand the importance and share a commitment to the division's second foundational pillar. From support staff to leadership, there has been a major change in the division's collective consciousness when it comes to supporting the advancement of diversity, inclusion, and equity throughout Oregon State University.

As managers of the university's messaging, URM has made steps toward shifting the symbolic dimension of oppression through critical attention to its brand, publications, social media presence, and communications to the university's constituents and the public. Through its commitments to sustained attention to issues of power and privilege, URM intends to continue this trajectory toward creating both a climate and culture within the division to foster inclusion, equity, and justice among its staff and a consistent message coming from the division that advances the goals of institutional transformation toward inclusion, equity, and justice. 
References

Bird, Sharon R. 2011. 'Unsettling Universities' Incongruous, Gendered Bureaucratic Structures: A Case-study Approach.” Gender, Work \& Organizations 18(2):202-230.

Bothwell, Michelle, Kali Furman, Qwo-Li Driskill, Rebecca Warner, Susan Shaw and Tuba Ozkän-Haller. 2018. "Empowering Faculty and Administrators to Re-Imagine a Socially Just Institution through Use of Critical Pedagogies," Proceedings of the $1^{\text {st }}$ Annual Conference of the Collaborative Network for Engineering and Computing Diversity Conference, Crystal City, VA.

Collins, P. H. (1993). Toward a new vision: Race, class and gender as categories of analysis and connection. Race, Sex and Class, 1 (1): 25-45.

Johnson Allan. Privilege, Power, and Difference, Third Edition. New York: McGraw-Hill, 2017.

May, Vivian. Pursuing Intersectionality, Unsettling Dominant Imaginaries. New York, NY: Routledge, 2015.

Morimoto, S. (2013). Beyond Binders Full of Women: NSF ADVANCE and Initiatives for Institutional Transformation. Sociological Spectrum, 33 (5): 397-415.

Oregon State University University Relations and Marketing. https://advance.oregonstate.edu/sites/advance.oregonstate.edu/files/advance_all_cohort_urm_pre sentation.pdf. Accessed December 18, 2018. 